\title{
Neutrino emissivity and bulk viscosity of iso-CSL quark matter in neutron stars
}

\author{
David B. Blaschke* and Jens Berdermann ${ }^{\dagger}$ \\ *Institute for Theoretical Physics, University of Wroclaw, 50-204 Wroclaw, Poland \\ Bogoliubov Laboratory for Theoretical Physics, JINR Dubna, 141980 Dubna, Russia \\ ${ }^{\dagger}$ DESY, Platanenallee 6, D-15738 Zeuthen, Germany
}

\begin{abstract}
We present results for neutrino emissivities and bulk viscosities of a two-flavor color superconducting quark matter phase with isotropic color-spin-locked (iso-CSL) single-flavor pairing which fulfill the constraints on quark matter derived from cooling and rotational evolution of compact stars. We compare with results for the phenomenologically successful, but yet heuristic $2 \mathrm{SC}+\mathrm{X}$ phase.
\end{abstract}

Keywords: neutrino cooling, bulk viscosity, dense quark matter, color superconductivity PACS: $04.40 . D g, 12.38 .-t, 26.60 .+c$

\section{INTRODUCTION}

Recently, astrophysical observational programmes monitoring compact stars (CS) have provided new, high-quality data for the static properties as well as the thermal and spin evolution which put tight constraints on the equation of state (EoS) and transport properties of dense matter in CS interiors [1]. In particular, circumstantial evidence for large masses and radii of compact stars [2, 3] suggests that the EoS at high densities must be rather stiff, possibly excluding quark matter interiors. In this debate, it has been demonstrated that modern EoS for quark matter allow for extended quark matter cores of CS while satisfying mass and radius constraints $[4,5,6,7]$. The resulting stiff hybrid star EoS suffers, however, from the masquerade problem [8]: the corresponding hybrid stars appear to have almost identical static properties when compared with pure neutron stars.

A diagnostic tool more sensible than the EoS are the thermal and transport properties of dense matter which determine, e.g., the cooling and spin evolution of CS. If quark matter occurs in CS interiors we expect it to be in a color superconducting state which entails a dramatic dependence on the pairing pattern and the sizes of pairing gaps. In this contribution, we will focus on the discussion of direct Urca neutrino emissivities and bulk viscosities of color superconducting quark matter.

The numerical analysis is based on a Nambu-Jona-Lasinio (NJL) type model, allowing a consistent determination of the density and temperature dependence of quark masses, pairing gaps and chemical potentials under neutron star constraints. The resulting phase diagram suggests that three-flavor phases of the CFL-type occur only at rather high densities [9, 10] and render hybrid star configurations gravitationally unstable $[11,5]$. Moreover, due to large pairing gaps in CFL quark matter, the r-mode instabilities cannot be damped [12] and cooling is inhibited [13]. 
Therefore, we will focus on two-flavor color superconducting phases in compact stars, the $2 \mathrm{SC}+\mathrm{X}$ phase [14], for which a detailed investigation of the cooling phenomenology for hybrid stars has already been worked out $[15,16]$, and the iso-CSL phase $[17,18]$ for which a consistent microscopic calculation of the direct Urca emissivity and the bulk viscosity will be presented here for the first time [19]. This will form the basis of further phenomenological applications in CS physics.

\section{QUARK MATTER IN THE ISO-CSL PHASE}

Single-flavor spin-one pairing channels have been investigated before, e.g., in [20, 21, 22]. We consider the iso-CSL phase $[17,18]$ for which in the pairing gap matrix $\hat{\Delta}=\Delta\left(\gamma_{3} \lambda_{2}+\gamma_{2} \lambda_{5}+\gamma_{1} \lambda_{7}\right)$ where the three antisymmetric color matrices $\left(\lambda_{2}, \lambda_{5}, \lambda_{7}\right)$ are locked to the three spin matrices $\left(\gamma_{3}, \gamma_{2}, \gamma_{1}\right)$. The two flavor channels are decoupled and the thermodynamical potential is therefore

$$
\Omega_{q}(T, \mu)=\sum_{f=u, d} \Omega\left(T, \mu_{f}\right)
$$

where the contribution of a single flavor in mean field approximation is given by

$$
\Omega\left(T, \mu_{f}\right)=\frac{\bar{\sigma}_{f}^{2}}{8 G_{S}}+\frac{3 \Delta_{f}^{2}}{8 G_{D}}-\sum_{r=1}^{6} \int \frac{d^{3} p}{(2 \pi)^{3}}\left[E_{f, r}(p)+2 T \ln \left(1+e^{-E_{f, r}(p) / T}\right)\right],
$$

The most important feature of the energy spectrum is that the dispersion relations of all modes $E_{f, r}$ have nonvanishing gaps and the lowest excitation energy is of $\mathscr{O}(1 \mathrm{MeV})$, as required from cooling phenomenology. Without a microscopic basis, such a mode spectrum has been postulated within the $2 \mathrm{SC}+\mathrm{X}$ phase [14]. In the following we compare results for direct Urca neutrino emissivities and bulk viscosities of these two phases, using the parameter set for constituent quark mass $M(p=0)=380 \mathrm{MeV}$ from Ref. [23].

\section{Neutrino emissivity}

Following Iwamotos seminal paper [24] where the direct Urca emissivity of quark matter,

$$
\varepsilon_{\mathrm{Urca}}^{v}=\frac{457}{630} \alpha_{s} G_{F}^{2} \mu_{e} \mu_{u} \mu_{d} T^{6}
$$

has first been derived, there have been a number of calculations, in particular for color superconducting phases. From the most recent ones beyond the exponential suppression ansatz, we refer to [25, 26, 27]). However, none of these is useable for cooling simulations because they have either ungapped modes which result in too fast cooling or the pairing pattern is not microscopically founded. Nevertheless, in deriving the neutrino emissivities for the $2 \mathrm{SC}+\mathrm{X}$ and the iso-CSL phase we follow the strategy of these Refs. 
with the result

$$
\begin{aligned}
\varepsilon_{2 \mathrm{SC}+\mathrm{X}}^{v} & =\varepsilon_{\mathrm{Urca}}^{v}\left[\frac{1}{3} e^{-\Delta_{X}(T)}+\frac{2}{3} G(\Delta)\right], \\
\varepsilon_{\mathrm{iso}-\mathrm{CSL}}^{v} & =\varepsilon_{\mathrm{Urca}}^{v} G\left(\Delta_{u}, \Delta_{d}\right),
\end{aligned}
$$

where we introduced the functions

$$
\begin{aligned}
G(\Delta) & =\frac{5040}{457 \pi^{6}} \int_{0}^{\infty} d z z^{3} \mathscr{F}(z) \quad(2 \text { SC Phase }) \\
G\left(\Delta_{u}, \Delta_{d}\right) & \left.=\frac{5040}{1371 \pi^{6}} \int_{0}^{\infty} d z z^{3}\left[\mathscr{F}_{1}(z)+\mathscr{F}_{3}(z)+\mathscr{F}_{5}(z)\right] \quad \text { (iso }- \text { CSL Phase }\right)(6)
\end{aligned}
$$

with

$$
\begin{aligned}
\mathscr{F}_{r}(z)=\sum_{e_{1}, e_{2}= \pm} \int_{0}^{\infty} \int_{0}^{\infty} d x d y & \left(e^{-e_{1} \sqrt{y^{2}+a_{u, r} \Delta_{u}^{2}}}+1\right)^{-1}\left(e^{e_{2} \sqrt{x^{2}+a_{d, r} \Delta_{d}^{2}}}+1\right)^{-1} \\
& \times\left(e^{z+e_{1} \sqrt{y^{2}+a_{u, r} \Delta_{u}^{2}}-e_{2} \sqrt{x^{2}+a_{d, r} \Delta_{d}^{2}}}+1\right)^{-1}
\end{aligned}
$$

characterising the influence of the superconducting gaps on the corresponding emissivity. The coefficients $a_{u, r}$ and $a_{d, r}$ for $r=1,3,5$ are defined in Ref. [17]. The function $\mathscr{F}(z)$ is given by (7) for the case $\Delta_{u}=\Delta_{d}=\Delta$ and $a_{u, r}=a_{d, r}=1$. The influence of the temperature dependence is taken into account by

$$
\Delta(T)=\Delta_{0} \sqrt{1-{\frac{T}{T_{c}}}^{\beta}},
$$

where one can find values for $\beta$ between 1-3.2 in the literature. In the following calculations we have used $\beta=1$. In Figure 1 we show the emissivities for the microscopic iso-CSL phase (right panel) in comaprison with the purely phenomenological $2 \mathrm{SC}+\mathrm{X}$ phase (left panel) as a function of temperature for different chemical potentials. For both phases a similar suppression of the emissivity is obtained. Hence the iso-CSL phase is probably able to explain recent cooling data in a more consistent way supporting the idea of superconducting phases in quark stars as explanation for observed fast CS cooling.

\section{Bulk viscosity}

According to [28], in the absence of viscosity all rotating CS would become unstable against r-modes [29]. Therefore, from the observation of millisecond pulsars, one can derive constraints for the composition of CS interiors [12,30]. For such an investigation, the bulk viscosity is a key quantity and we want to consider it for the two-flavor color superconducting phases introduced above, following the approach described in Ref. [31]. Note that the 2SC phase considered in [32] and in the contribution to this volume 

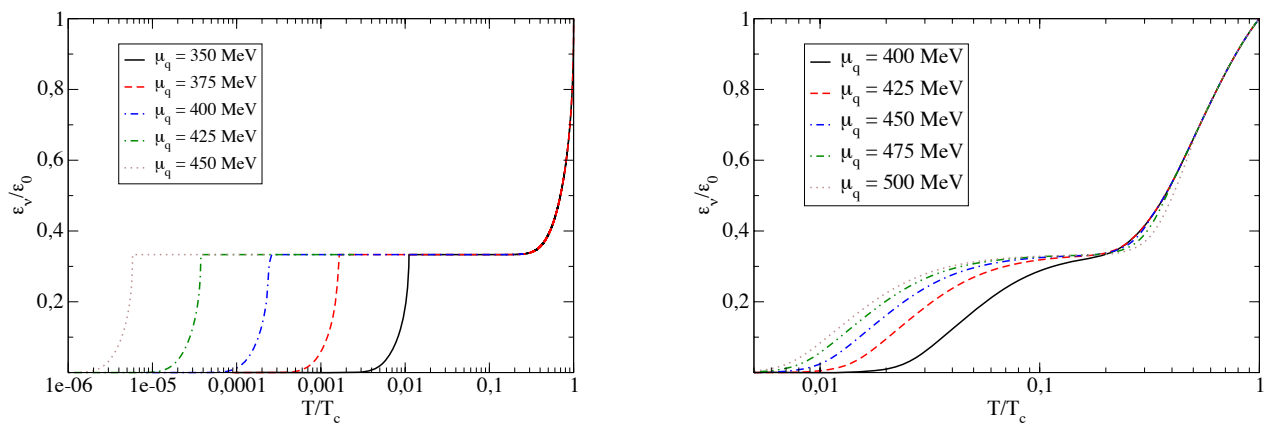

FIGURE 1. Neutrino emissivities due to direct Urca processes in the $2 \mathrm{SC}+\mathrm{X}$ phase (left) and in the iso-CSL phase (right).

[33] is a three-flavor phase, where the nonleptonic process $u+d \leftrightarrow u+s$ provides the dominant contribution. Due to absence of strange quarks in the 2SC phase of the present paper, this process does not occur.

The bulk viscosity at all temperatures is determined by

$$
\zeta=\frac{\lambda C_{t}^{2}}{\omega^{2}+(\lambda B / n)^{2}}
$$

with $C_{t}=C+C^{6}$ and the coefficients functions

$$
\begin{aligned}
C & =\frac{M_{u}^{2}}{3 \mu_{u}}-\frac{M_{d}^{2}}{3 \mu_{d}} \\
C^{6} & =\frac{4 \alpha_{s}}{3 \pi}\left[\frac{M_{d}^{2}}{\mu_{d}}\left(\ln \frac{2 \mu_{d}}{M_{d}}-\frac{2}{3}\right)-\frac{M_{u}^{2}}{\mu_{u}}\left(\ln \frac{2 \mu_{u}}{M_{u}}-\frac{2}{3}\right)\right] \\
B & \simeq \frac{\pi^{2}}{3} n\left(\frac{1}{\mu_{u}^{2}}+\frac{1}{\mu_{d}^{2}}+\frac{1}{\mu_{e}^{2}}\right) .
\end{aligned}
$$

The relevant processes for the bulk viscosity in two-flavor quark matter are the flavor changing weak processes of electron capture and beta decay, with a direct relation to the direct Urca emissivity

$$
\begin{aligned}
\lambda_{2 \mathrm{SC}+\mathrm{X}} & =\frac{714}{457 \pi^{2} T^{2}} \varepsilon_{\mathrm{Urca}}^{v}\left[\frac{1}{3} e^{-\Delta_{X}(T)}+\frac{2}{3} H(\Delta)\right], \\
\lambda_{\text {iso-CSL }} & =\frac{714}{457 \pi^{2} T^{2}} \varepsilon_{\text {Urca }}^{v} H\left(\Delta_{u}, \Delta_{d}\right),
\end{aligned}
$$

where $H(\Delta)=\frac{457 \pi^{2}}{1428} G(\Delta)$ and analogously for $H\left(\Delta_{u}, \Delta_{d}\right)$. The numerical results for the NJL model in selfconsistent meanfield approximation are displayed in Fig. 2 for the 
2SC+X phase (left panel) and the iso-CSL phase (right panel) in striking similarity. Note that in comparison with Ref. [31] the peak value of the viscosity is also located at $T=1 \sim 2 \mathrm{MeV}$, but up to three orders of magnitude higher! Since the normal quark matter results coincide, this must be a result of the selfconsistent treatment of masses, gaps and composition (chemical potentials) in the present models.
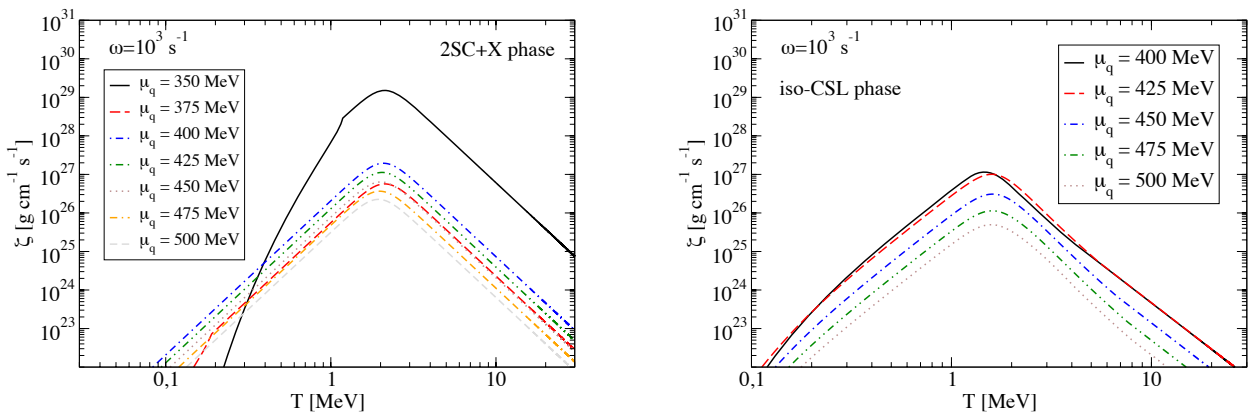

FIGURE 2. Temperature dependence of bulk viscosities in the $2 \mathrm{SC}+\mathrm{X}$ phase (left) and in the iso-CSL phase (right) for a frequency of $\omega=1 \mathrm{kHz}$, typical for excitations of r-modes in millisecond pulsars.

\section{CONCLUSION}

Transport properties in dense quark matter depend sensitively on the color superconductivity pairing patterns and provide thus a tool for unmasking the CS interiors by their cooling and rotational evolution characteristics. On the example of neutrino emissivities and bulk viscosities for the $2 \mathrm{SC}+\mathrm{X}$ and the iso-CSL phase we have demonstrated that both two-flavor color superconducting phases fulfill constraints from the CS phenomenology. For the $2 \mathrm{SC}+\mathrm{X}$ phase with yet heuristic assumptions for the X-gap the hybrid star configurations and their cooling evolution have been numerically evaluated in accordance with observational data. The temperature and density behavior of the neutrino emissivity in the microscopically well-founded iso-CSL phase appear rather similar so that we expect a good agreement with CS cooling data too. The bulk viscosities for both phases have been presented here for the first time and provide sufficient damping of r-mode instabilities to comply with the phenomenology of rapidly spinning CS. We conclude that the subtle interplay between suppression of the direct Urca cooling process on the one hand and sufficiently large bulk viscosity puts severe constraints on microscopic approaches to quark matter in compact stars. 


\section{ACKNOWLEDGMENTS}

D.B. likes to thank M. Alford, R. Anglani, A. Drago, B. A. Sa'd and H. Malekzadeh for discussions and is grateful to the organizers for the invitation and for the stimulating atmosphere of the meeting. He received support from the Polish Ministry for Science and Higher Education.

\section{REFERENCES}

1. T. Klähn et al., Phys. Rev. C 74, 035802 (2006).

2. J. E. Trümper, V. Burwitz, F. Haberl and V. E. Zavlin, Nucl. Phys. Proc. Suppl. 132, 560 (2004)

3. F. Özel, Nature 441, 1115 (2006).

4. M. Alford, D. Blaschke, A. Drago, T. Klähn, G. Pagliara and J. Schaffner-Bielich, Nature 445, E7 (2007).

5. T. Klähn et al., Phys. Lett. B 654, 170 (2007).

6. D. B. Blaschke, D. Gomez Dumm, A. G. Grunfeld, T. Klähn and N. N. Scoccola, Phys. Rev. C 75, 065804 (2007).

7. A. G. Grunfeld, J. Berdermann, D. B. Blaschke, D. Gomez Dumm, T. Klähn and N. N. Scoccola, Int. J. Mod. Phys. E in press; arXiv:0705.3787 [hep-ph], (2007).

8. M. Alford, M. Braby, M. W. Paris and S. Reddy, Astrophys. J. 629, 969 (2005).

9. S. B. Ruester, V. Werth, M. Buballa, I. A. Shovkovy and D. H. Rischke, Phys. Rev. D 72, 034004 (2005).

10. D. Blaschke, S. Fredriksson, H. Grigorian, A. M. Oztas and F. Sandin, Phys. Rev. D 72, 065020 (2005).

11. M. Buballa, Phys. Rep. 407, 205 (2005).

12. J. Madsen, Phys. Rev. Lett. 85, 10 (2000)

13. D. Blaschke, T. Klähn and D. N. Voskresensky, Astrophys. J. 533, 406 (2000).

14. H. Grigorian, D. Blaschke and D. Voskresensky, Phys. Rev. C 71, 045801 (2005).

15. S. Popov, H. Grigorian and D. Blaschke, Phys. Rev. C 74, 025803 (2006).

16. D. Blaschke and H. Grigorian, Prog. Part. Nucl. Phys. 59, 139 (2007).

17. D. N. Aguilera, D. Blaschke, M. Buballa, and V. L. Yudichev, Phys. Rev. D 72, 034008 (2005).

18. D. N. Aguilera, D. Blaschke, H. Grigorian, and N. N. Scoccola, Phys. Rev. D 74, 114005 (2006).

19. J. Berdermann and D. Blaschke, in preparation (2007).

20. T. Schäfer, Phys. Rev. D 62, 094007 (2000).

21. M. G. Alford, J. A. Bowers, J. M. Cheyne and G. A. Cowan, Phys. Rev. D 67, 054018 (2003).

22. A. Schmitt, Phys. Rev. D 71, 054016 (2005).

23. H. Grigorian, Phys. Part. Nucl. Lett. 4 (2007).

24. N: Iwamoto, Ann. Phys. 141, 1 (1982).

25. P. Jaikumar, C. D. Roberts and A. Sedrakian, Phys. Rev. D 73, 042801 (2006).

26. A. Schmitt, I. A. Shovkovy and Q. Wang, Phys. Rev. D 73, 034012 (2006).

27. Q. Wang, Z. Wang and J. Wu, Phys. Rev. D 74, 014021 (2006).

28. N. Andersson, Astrophys. J. 502, 708 (1998).

29. N. Andersson and K. D. Kokkotas, Int. J. Mod. Phys. D 10, 381 (2001).

30. A. Drago, G. Pagliara and I. Parenti, arXiv:0704.1510 [astro-ph] (2007).

31. B. A. Sa'd, I. A. Shovkovy and D. H. Rischke, Phys. Rev. D 75, 065016 (2007).

32. M. G. Alford and A. Schmitt, J. Phys. G 34, 67 (2007).

33. M. G. Alford and A. Schmitt, arXiv:0709.4251 [nucl-th] (2007). 